\title{
Treatment Effects of Ultrasound Guide Selective Nerve Root Block for Lower Cervical Radicular Pain: A Retrospective Study of 1-Year Follow-up
}

\author{
Yongbum Park, $\mathrm{MD}^{1}$, Jae Ki Ahn, $\mathrm{MD}^{1}$, Yulkyung Sohn, $\mathrm{MD}^{1}$, \\ Haemi Jee, $\mathrm{PhD}^{2}$, Ji Hae Lee, $\mathrm{MD}^{3}$, Jongwoo Kim, $\mathrm{MD}^{4}$, Ki Deok Park, $\mathrm{MD}^{5}$
}

\begin{abstract}
${ }^{1}$ Department of Rehabilitation Medicine, Sanggye Paik Hospital, Inje University College of Medicine, Seoul; ${ }^{2}$ Department of Health \& Fitness Management, Namseoul University, Cheonan; Departments of ${ }^{3}$ Radiology and ${ }^{4}$ Family Medicine, Sanggye Paik Hospital, Inje University College of Medicine, Seoul; ${ }^{5}$ Department of Rehabilitation Medicine, Gachon University Gil Medical Center, Incheon, Korea
\end{abstract}

Objective To compare the long-term effects and advantages of ultrasound (US)-guided selective cervical nerve root block with fluoroscopy (FL)-guided transforaminal block.

Methods From March 2009 to November 2012, 162 patients received steroid injections for lower cervical radicular pain. A total of 114 patients fulfilled the inclusion criteria. All procedures were performed by using US or FL. We compared the intravascular injections during the procedure with the effects and functional scales at 3, 6, and 12 months after the procedure between the two groups. Successful treatments occurred when patients obtained significant pain reliefs (as measured by $>50 \%$ improvements in the verbal numerical scale [VNS] score and $>40 \%$ improvements in the neck disability index [NDI] score) and reported a patient satisfaction score of 3 or 4 points at 12 months after the injection. Image analysis of intravascular injection and chart review were performed. Logistic regression was performed to reveal the correlations between successful treatments and variables (patient's age, gender, duration of the disease, cause, injection method, and radiologic finding).

Results The VNS and NDI improved 3 months after the injection and continued to improve until 12 months for both groups. But there were no statistical differences in changes of VNS, NDI, and effectiveness between these two groups. The proportion of patients with successful treatment is illustrated as $62.5 \%$ in US-guided group and $58 \%$ in FL-guided group at 12 months. There were no significant differences between the groups or during follow-up periods. Three cases of the intravascular injections were done in FL-guided group.

Conclusion The US-guided selective cervical nerve root blocks are facilitated by identifying critical vessels at unexpected locations relative to the foramen and to protect injury to such vessels, which is the leading cause of reported complications from FL-guided transforaminal blocks. On treatment effect, significant long-term improvements in functions and pain reliefs were observed in both groups after the intervention. However, significant differences were not observed between the groups. Therefore, the US-guided selective cervical nerve root block was shown to be as effective as the FL-guided transforaminal block in pain reliefs and functional improvements, in addition to the absence of radiation and protection vessel injury at real-time imaging.

Keywords Ultrasonography, Fluoroscopy, Cervical radiculopathy, Nerve block

Received March 4, 2013; Accepted June 25, 2013

Corresponding author: Ki Deok Park

Department of Rehabilitation Medicine, Gachon University Gil Medical Center, 21 Namdong-daero 774beon-gil, Namdong-gu, Incheon 405-760, Korea Tel: +82-32-460-8374, Fax: +82-32-460-3051, E-mail: bduck@gachon.ac.kr

(c) This is an open-access article distributed under the terms of the Creative Commons Attribution Non-Commercial License (http://creativecommons.org/ licenses/by-nc/3.0) which permits unrestricted noncommercial use, distribution, and reproduction in any medium, provided the original work is properly cited. Copyright $\odot 2013$ by Korean Academy of Rehabilitation Medicine 


\section{INTRODUCTION}

Epidural steroid injection is considered to reduce inflammatory edema of the injured nerve roots, decrease sensitization of the dorsal horn neurons, and suppress the transmission of nociceptive C-fibres [1]. Two principal techniques are available in order to deliver medication into the cervical epidural space: the transforaminal and interlaminar routes. The transforaminal approach is advantageous because corticosteroid preparations can be closely injected to the probable source of the irritated nerve root, and this approach results in better ventral epidural spreading than the interlaminar approach $[2,3]$.

Although computer tomography (CT) or fluoroscopy (FL) guidance is the standard aiding procedure for transforaminal approach, these techniques are timeconsuming and involve radiation exposure. On the other hand, ultrasound (US) dose not expose the patients or personnel to radiations and the image can be performed continuously during the injection.

Narouze et al. [4] demonstrated that US-guided cervical selective nerve root block allowed the needle to be located within $5 \mathrm{~mm}$ of the targeted location. Moreover, the US-guided approach was reported to be a safe procedure since the blood vessels near the targeted area could be avoided with continuous visualization on the real-time images of the treatment site [4]. Yamauchi et al. [5] reported on the improvement in symptoms at 24 hours and 1 month after the US-guided approach.

Above studies have not yet been performed to compare the US with other methods, such as FL. Therefore, there are limitations to propose the usefulness of injection methods by using US. Jee et al. [6] compared the shortterm treatment effects between US-guided cervical selective nerve root blocks and FL-guided cervical transforaminal epidural blocks, and significant differences were not observed between the groups. Unlike injections using FL, the contrast agent often reflexes from proximal spinal canal and epidural space to distal portion in US-guided approach after injection. For such reasons, treatment effects could not last long-term since medications cannot be injected to lesion sites in some cases. Therefore, the effects of injections between US and FL need to be compared by long-term follow-ups.

The aims of this study are to compare the treatment effects of US-guided cervical selective nerve root block and
FL-guided cervical transforaminal epidural block for one year in patients with lower cervical radicular pain and to identify factors influencing the treatment results of injections.

\section{MATERIALS AND METHODS}

\section{Subjects}

This study included 162 patients who underwent either US-guided cervical selective nerve root block or FL-guided cervical transforaminal epidural block due to lower cervical radicular pains from March 2009 to November 2012. This study involved patients who were suspected of cervical radicular pain based on physical examination, inquiry or electromyography, and also patients who were confirmed with herniated cervical intervertebral disc (HCD) and spinal stenosis in cervical CT and magnetic resonance imaging in corresponding nerve root location. Exclusion criteria were for history of cervical spinal surgery, cervical spondylotic myelopathy due to ossification of the posterior longitudinal ligament or central spinal stenosis, invasion in more than two nerve roots and association of segmental instability.

A total of 114 subjects satisfied the inclusion criteria and were divided into US-guided group of 64 patients and FL-guided group of 50 patients. US-guided group comprised of 24 males and 40 females, and FL-guided group comprised of 21 males and 29 female. Mean ages were 57.1 years old in US-guided group, 56.0 years old in FL-guided group. Mean duration of therapeutic injections after symptom expression was 6.8 weeks in USguided group and 6.7 weeks in FL-guided group. As causes of cervical radicular pain, 11 subjects had HCD and 53 subjects had spinal stenosis in US-guided group, 7 subjects had HCD and 43 subjects had spinal stenosis in FL-guided group. Injection sites were C5 in 9 subjects, C6 in 40 subjects, and C7 in 15 subjects for US-guided group and C5 in 4 subjects, C6 in 35 subjects, and C7 in 11 subjects for FL-guided group.

The patients received 2 consecutive therapeutic injections with a two-week interval between the injections. The second injection proceeded conditionally. If the initial injection resulted in significant symptom reduction (verbal numerical rating scale $[\mathrm{VNS}] \geq 50 \%$ ), the second injection was omitted with a progression follow-up. If no pain reliefs or pain deteriorations were observed, a sec- 
Table 1. Characteristics of the patients

\begin{tabular}{|c|c|c|c|}
\hline & Ultrasound-guided $(n=64)$ & Fluoroscopy-guided $(n=50)$ & p-value \\
\hline Age (yr) & $57.1 \pm 9.5$ & $56.0 \pm 9.4$ & 0.704 \\
\hline \multicolumn{4}{|l|}{ Gender } \\
\hline Male & $24(37.5)$ & $21(42.0)$ & \\
\hline Female & $40(62.5)$ & $29(58.0)$ & 0.626 \\
\hline $\operatorname{BMI}\left(\mathrm{kg} / \mathrm{m}^{2}\right)$ & $23.5 \pm 2.0$ & $23.5 \pm 1.8$ & 0.529 \\
\hline Duration (mo) & $6.8 \pm 2.1$ & $6.7 \pm 2.1$ & 0.919 \\
\hline No. of injection & $1.5 \pm 0.6$ & $1.7 \pm 0.6$ & 0.934 \\
\hline \multicolumn{4}{|l|}{ Cause } \\
\hline HCD & $11(17.2)$ & $7(14.0)$ & \\
\hline Steosis & $53(82.8)$ & $43(86.0)$ & 0.643 \\
\hline \multicolumn{4}{|l|}{ Target root } \\
\hline C5 & $9(14.1)$ & $4(8.0)$ & \\
\hline C6 & $40(62.5)$ & $35(70.0)$ & \\
\hline $\mathrm{C} 7$ & $15(23.4)$ & $11(22.0)$ & 0.557 \\
\hline
\end{tabular}

Values are presented as mean \pm standard deviation or number (\%).

BMI, body mass index; HCD, herniated cervical disc.

ond injection or reevaluation was not considered. Such patients were withdrawn from the study without subsequent data collection since the cause of the pain was considered outside the inclusion criteria. If the patients experienced pain reliefs of less than $50 \%$ reduction in VNS, a second injection was scheduled with a progression follow-up. No statistical differences were found in injection site, cause of pain, duration, gender, age, and body mass index (BMI) between two groups (Table 1).

\section{Injection method}

\section{US-guided selective nerve root block}

All treatments were performed as an outpatient procedure. Accuvix XQ (Medison, Seoul, Korea) with a linear probe at 6 to $12 \mathrm{MHz}$ was used as the US instrument [47]. With the patient's head externally rotated at $30^{\circ}$ to $40^{\circ}$ away from the targeted area in supine position, the frontal cervical spine area from the clavicle to mandible was adequately disinfected with Betadine and an aseptic dressing was applied. The sternocleidomastoid muscle was first palpated from the posterior side to palpate the transverse process of the cervical spine. A probe was placed and a transverse scan image of the transverse process was obtained after locating the transverse process. The location of the 7th transverse process was confirmed by probing adjacent transverse processes from the superior to the inferior direction. Unlike the transverse processes of the 3rd to 6th cervical spines, the transverse process of the 7th cervical spine has a rudimentary anterior tubercle and a prominent posterior tubercle [7].

The targeted transverse process was identified by slowly moving the probe in all directions with the 7th cervical spine transverse process as the reference point. The optimal image of the nerve root, the location of the radicular artery, and the surrounding vessels near the border of the nerve root were obtained through the probe manipulation. Next, a spine needle 22-G Spinocan (B. Braun, Melsungen, Germany) was inserted from the posterior to anterior direction, towards the nerve root and parallel to the probe. The needle end was placed on the dorsal side of the nerve by cautiously avoiding damages to the possible deep cervical artery near the insertion site and thus, locating the area free of the radicular artery. First, $1 \mathrm{~mL}$ of the contrast media Omnipaque 300 (GE Healthcare, Carrigtwohill, Ireland) was injected. The anteroposterior images were obtained to confirm the distribution or spread pattern of the injected contrast media with C-arm FL. The following steps were initiated after confirming for proper shadowed contrasts of the nerve root and the absence of intravascular injection of the contrast media. The $1 \mathrm{~mL}$ of $1 \%$ lidocaine was injected and monitored for the onset of clinical manifestations, such as midneck and contralateral arm pain, metallic taste, dizziness, tachycardia, full body paresthesias, auditory changes, slurred 
speech, and motor ataxia for 1 to 2 minutes [8]. The $3 \mathrm{~mL}$ of the treatment drug, composed of $2 \mathrm{~mL}$ dexamethasone (10 mg) and $1 \mathrm{~mL} 0.5 \%$ lidocaine, was injected after confirming the absence of abnormal findings (Fig. 1).

\section{FL-guided transforaminal epidural block}

The transforaminal epidural injection was conducted under the FL-guidance by the same physician. All treatments were performed as an outpatient procedure. With the patient's head externally rotated $30^{\circ}$ to $40^{\circ}$ away from the procedure site in a supine position, the frontal cervical spine area from the clavicle to mandible was adequately disinfected with Betadine and the aseptic dress- ing was applied.

The fluoroscopic apparatus KMC 950 (KOMED, Gwangju, Korea) was adjusted to obtain a proper oblique and well-defined view of the intervertebral foramen. After applying a local anesthesia with $1 \%$ lidocaine to the targeted area, the distal $5 \mathrm{~mm}$ end of the spine needle 22-G Spinocan (B. Braun) was bent about $15^{\circ}-20^{\circ}[9]$. The angulated needle end was carefully tilted towards the posterior side and contacted the medial side of the superior articular process, which composes the posterior side of the targeted foramen. When the needle end contacted the superior articular process, the needle was turned $180^{\circ}$ proceeding 2 to $3 \mathrm{~mm}$ in the forwarding di-
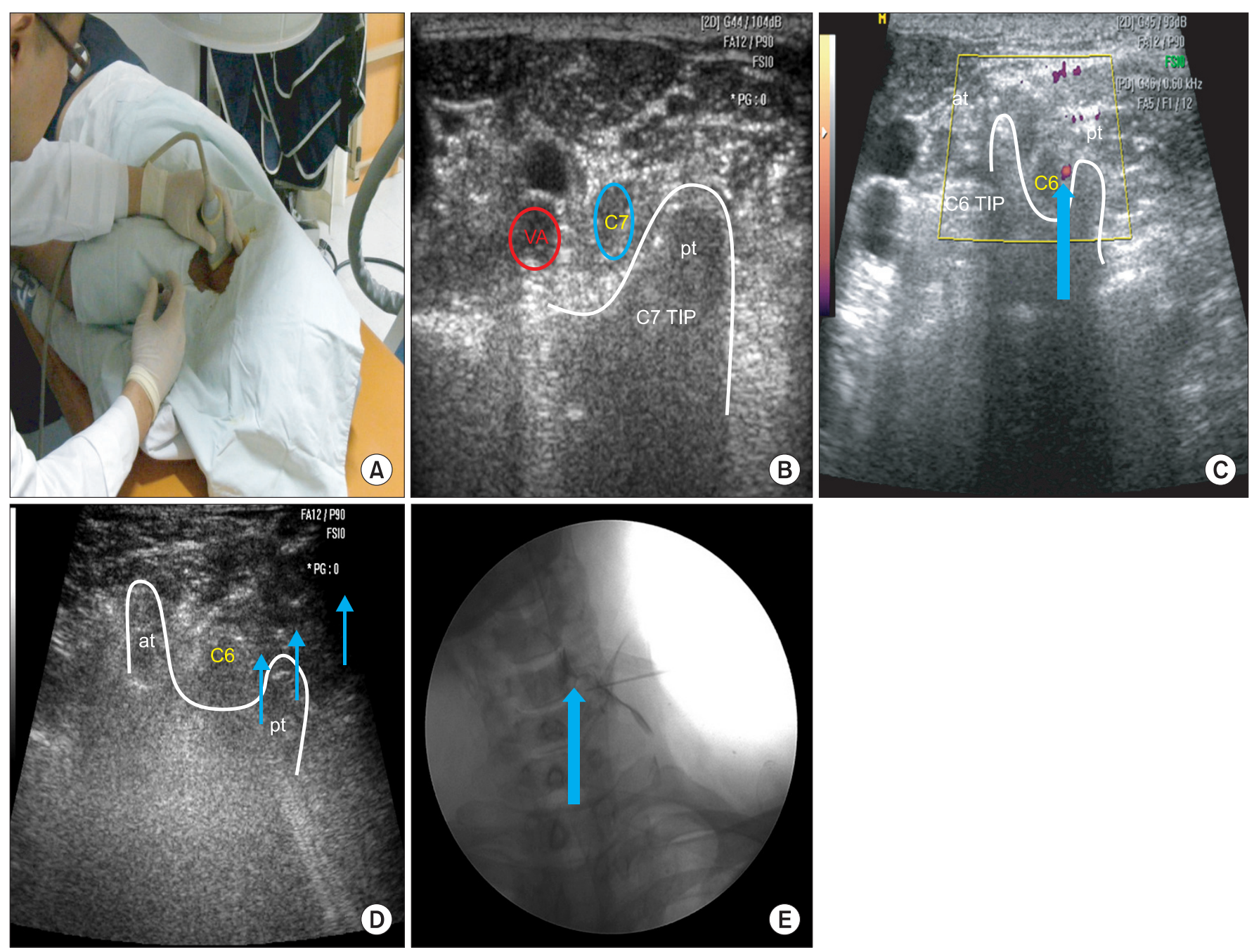

Fig. 1. (A) Ultrasound-guided selective nerve root block (target nerve root C6). (B) This figure shows axial transverse ultrasound image of the $\mathrm{C} 7$ transverse process which has only 1 posterior tubercle. (C) Power Doppler image shows radicular arterial blood flows (arrowhead) of the C6 nerve root. (D) Needle (arrows) is placed on the dorsal surface of the C6 nerve root. (E) The targeted C6 nerve root is outlined by $1-2 \mathrm{~mL}$ contrast media (arrow). Contrast media spread to intraforaminal lesion (arrowhead). AT, anterior tubercle; PT, posterior tubercle. 
rection under continuous FL-guidance. The depth of the fluoroscopic apparatus was adjusted under the anteroposterior view to locate the needle end at the center of the articular pillar. The cerebrospinal fluid and blood absorption tests were conducted to detect possible blood traces in non-targeted areas. The fluoroscopic images were continuously monitored while injecting non-ionic contrast media Omnipaque 300 (GE Healthcare) to confirm the shadowed contrast of the foramen, nerve root, epidural space, and other related structures. The $1 \mathrm{~mL}$ of $1 \%$ lidocaine was injected if the needle was repositioned for vessel infusion, the images of the nerve root showed properly shadowed contrast, and the contrast media was not identified within any proximal vessel. The patients were monitored for the onset of clinical manifestations, such as midneck and contralateral arm pain, metallic taste, dizziness, tachycardia, full body paresthesias, auditory changes, slurred speech, and motor ataxia for 1 to 2 minutes after the injection [8]. After confirming the absence of abnormal findings, the FL-guided approach was completed after injecting the drug similar to the USguided approach.

Treatment effects between the approaches and correlation between the treatment effects and variables After retrospectively reviewing medical records, we classified the cause of cervical radicular pain as being due to spinal stenosis or HCD. The patient's demographic variables were also detailed at the initial evaluation. In USguided approach, the injection of medication to the intraspinal epidural space or distal portion was confirmed based on radiographic imaging. The degree of pain improvement was measured with VNS as to compare the effects of injection. The degree of functional improvement was identified with neck disability index (NDI). When patient record was unidentifiable during the follow-up, injection method was inquired over the telephone by an independent investigator. Patients were surveyed with 1) re-injection or operation and 2) VNS, NDI, and patient's satisfaction. The effect of treatment was considered as failure in cases of re-injection and surgical procedures. VNS and NDI were confirmed for statistical analysis and excluded afterwards.

When using VNS, the patients were asked to rate their pains on a scale from zero to 10 , where zero and 10 represented "no pain" and "the worst pain possible," respec- tively, in whole numbers with 11 integers including zero [10]. A medical investigator marked the scale for patients who were incapable of marking for themselves by directing and slowly moving from zero to 10 on a VNS scale board. The patients knotted at the appropriate score.

The degree of physical disability was measured with NDI, which is the most widely used survey questionnaire assessing cervical spine abnormality. NDI was first developed to evaluate the degree of limitations in daily lives of patients with severe cervical pains, especially for those with whiplash trauma [11]. NDI with 10 questionnaires is composed of 7 functional activity-related, 2 symptomrelated, and 1 concentration-related questions. The final NDI score was obtained by adding all scores from the questions. Higher NDI score indicated increased functional disability related to cervical abnormality. The original developer, Vernon, suggested interpreting the scores by the following ranges: 4 or lower, no disability; 5 to 14 , mild disability; 15 to 24 , moderate disability; 25 to 34 , severe disability; 35 or over, complete disability.

The patient satisfactory scores were measured with a 5 -grade scale ( $<0$, no effect at all; 1 , bad; 2 , fair; 3 , good; $\geq 4$, excellent) at 12 months after the treatments. 'Excellent' meant satisfied with the treatment result as expected, 'good' meant not as much as expected but willing to try this treatment next time when pain redevelops, 'fair' meant had some effects but not enough to choose the same treatment next time when pain re-develops, and finally, 'bad' meant same effects prior to the treatment or worse.

The degree of contrast agent diffusion was examined by taking X-ray from anteroposterior and lateral view for both US and FL approaches. Based on radiologic findings, the diffusion degree was divided into 'delivery to intraspinal epidural' or 'proximal foraminal lesion and distal portion' according to the diffusions of contrast agent to targeted nerve roots by a radiologist who was blinded with injection methods (Fig. 2). When VNS and NDI improved by more than $50 \%$ and $40 \%$, respectively, after every 3, 6, and 12 months, we considered treatment effect as successful [12]. Surgical treatment and re-injection due to relapse of symptom (VNS by $>50 \%$ ) in every period were defined failures.

Successful treatment occurred when patients obtained significant pain reliefs (as measured by $>50 \%$ improvement in the VNS score and $>40 \%$ improvement in the 

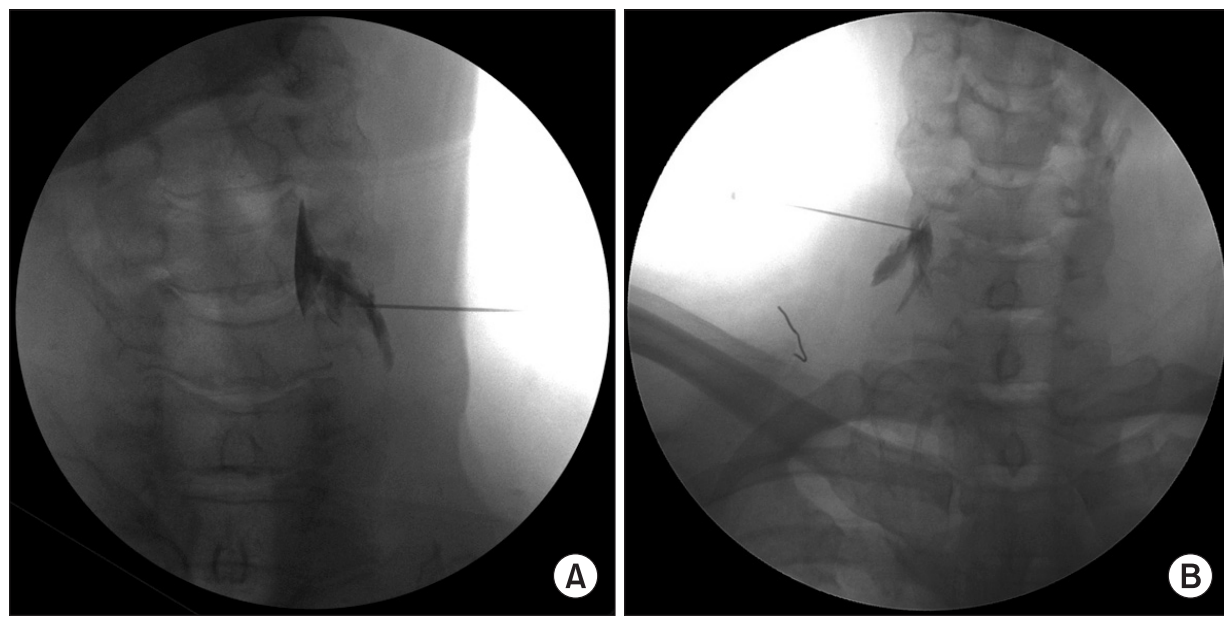

Fig. 2. Anteroposterior view for C6 ultrasound-guided selective C6 nerve root block. (A) Contrast spread to intraforaminal epidural space. (B) Contrast spread to only distal nerve root.

Table 2. Pain improvements of steroid injections with ultrasound- and fluoroscopy-guided at 3-, 6-, and 12-month follow-up assessments

\begin{tabular}{lcc}
\hline & $\begin{array}{c}\text { Ultrasound-guided } \\
(\mathbf{n}=64)\end{array}$ & $\begin{array}{c}\text { Fluoroscopy-guided } \\
(\mathbf{n}=\mathbf{5 0})\end{array}$ \\
\hline Baseline & $6.50 \pm 0.89$ & $6.34 \pm 0.82$ \\
$3 \mathrm{mo}$ & $2.75 \pm 1.05^{\mathrm{a})}$ & $2.60 \pm 1.11^{\mathrm{a})}$ \\
$6 \mathrm{mo}$ & $1.95 \pm 1.28^{\mathrm{a})}$ & $1.86 \pm 1.23^{\mathrm{a})}$ \\
$12 \mathrm{mo}$ & $1.42 \pm 1.22^{\mathrm{a})}$ & $1.36 \pm 1.27^{\mathrm{a})}$ \\
\hline
\end{tabular}

Values are presented as mean \pm standard deviation.

${ }^{a)} \mathrm{p}<0.05$ comparison of verbal numerical scale score with baseline.

NDI score) and reported a patient satisfaction score of 3 or 4 points at 12 weeks after the injections. In addition, we examined the effects of injection method, patient's age, gender, symptom, illness duration, cause of cervical nerve root pain, and degree of contrast agent diffusion on treatment success.

\section{Statistics}

Chi-square tests, Fisher exact tests, and Mann-Whitney $\mathrm{U}$ tests were used to compare differences in sex, age, BMI, target nerve root, cause of radicular pain, and duration of diseases between the two groups. At each time point, the VNS and NDI scores were compared when using repeated measures analysis of variance, and Bonferroni corrections were utilized for post hoc comparisons.

Patient satisfaction scores were analyzed with a chisquare test. The treatment outcome and variables (injection method, patient's age, gender, duration of the disease, and cause) were analyzed with logistic regres-
Table 3. Functional improvement of steroid injections with ultrasound- and fluoroscopy-guided at 3-, 6-, and 12-month of follow-up on assessments

\begin{tabular}{lcc}
\hline & $\begin{array}{c}\text { Ultrasound-guided } \\
(\mathbf{n}=\mathbf{6 4})\end{array}$ & $\begin{array}{c}\text { Fluoroscopy-guided } \\
(\mathbf{n}=\mathbf{5 0})\end{array}$ \\
\hline Baseline & $24.52 \pm 5.78$ & $24.76 \pm 5.96$ \\
$3 \mathrm{mo}$ & $16.97 \pm 4.06^{\mathrm{a})}$ & $16.96 \pm 5.21^{\mathrm{a})}$ \\
$6 \mathrm{mo}$ & $9.88 \pm 6.31^{\mathrm{a})}$ & $9.72 \pm 6.10^{\mathrm{a})}$ \\
$12 \mathrm{mo}$ & $7.22 \pm 5.20^{\mathrm{a})}$ & $6.28 \pm 5.31^{\mathrm{a})}$ \\
\hline
\end{tabular}

Values are presented as mean \pm standard deviation.

${ }^{a)} \mathrm{p}<0.05$ comparison of neck disability index score with baseline.

sion analyses. All analyses were performed with SAS Enterprise Guide 4.1 (SAS Institute, Cary, NC, USA) with a significance level of $\mathrm{p}<0.05$.

\section{RESULTS}

\section{Treatment effect between approaches}

No differences were shown between two groups in NDI and VNS scores before injection, as well as in treatment effects after injection at 3, 6, and 12 months, respectively (Tables 2, 3). In terms of period, 13 subjects underwent re-injection and a subject received operation at 3 months in US-guided group. Treatment was successful in 50 patients $(78.1 \%)$. Meanwhile, 10 subjects underwent re-injection and two subjects received operation at 3 months in FL-guided group. Treatment was successful in 38 patients (76\%). On the 6 months, 7 subjects underwent re-injection and 43 subjects $(67.2 \%)$ showed successful outcomes in US-guided group, and 5 subjects underwent 
re-injection and 33 subjects (66\%) showed successful outcomes in FL-guided group. On the 12 months, only 3 patients underwent re-injection and a total of 40 patients (62.5\%) showed successful results in US-guided group, and only 4 patients underwent re-injection and a total of 29 patients (58\%) showed successful results in FL-guided group (Fig. 3). No difference was found in the successful rate of treatment in every assessment period between two groups.

\section{Correlation between the treatment effects and variables}

According to radiographic analysis results, contrast agent was observed only in distal portion of 45 subjects (70.3\%) and flowed into intraspinal epidural space in 19 subjects $(29.7 \%)$ of US-guided group. Contrast medium flowed into intraspinal epidural space in all patients of FL-guided group.

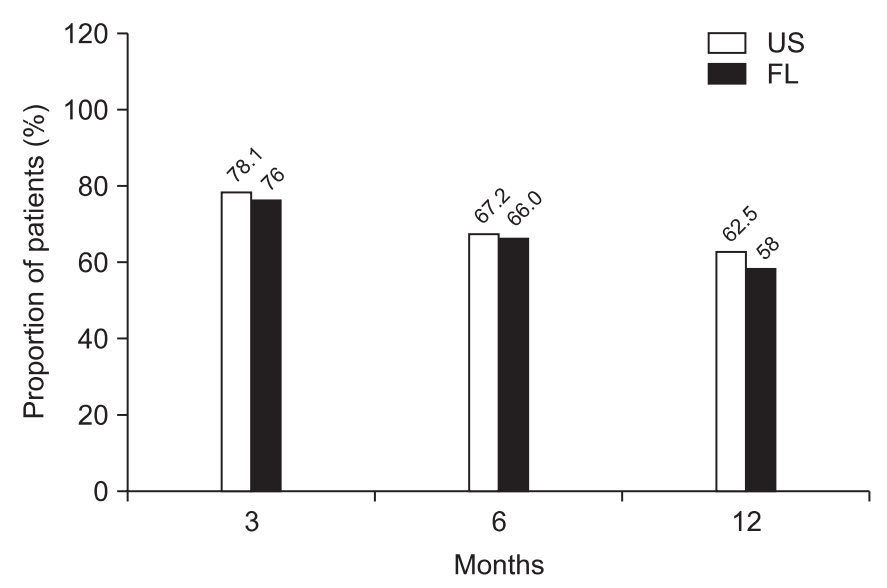

Fig. 3. Illustration of significant pain reliefs ( $\geq 50 \%$ reduction in verbal numerical scale from baseline) and functional improvements ( $\geq 40 \%$ reduction in neck disability index from baseline). US, ultrasound; FL, fluoroscopy.
Successful treatment occurred when patients obtained significant pain reliefs (as measured by $>50 \%$ improvement in the VNS score and $>40 \%$ improvement in the NDI score) and reported a patient satisfaction score of 3 or 4 points at 12 months after the injections. Treatment success was unrelated to either the US-guided nor the FL-guided approach, as shown in the cause of radicular pain, the patients' age and gender, the duration of symptoms, and contrast patterns (Table 4).

\section{Complications}

Three cases of vessel injections were only observed in the FL-guided transforaminal approach without a significant difference between the approaches ( $p>0.05)$. Complications, such as a dural puncture did not occur during both approaches. The patients with complications were transferred to the recovery room for observations. All symptoms disappeared prior to the discharge.

\section{DISCUSSION}

Cervical epidural steroid injections can be categorized by the needle insertion pathway into the epidural space and include the interlaminar and transforaminal approaches. Compared with an interlaminar epidural steroid injection, transforaminal approaches are expected to provide better delivery of medications to the site of radiculopathy and increased distribution of the drug into the ventral epidural space [13]. Although FL-guided cervical nerve root block is a widely known standard procedure, accidental intravascular injections can lead to catastrophic problems, such as vertebral artery dissection or spinal cord infarction [14-16].

In recent years, several studies presented the usefulness of US in cervical selective nerve root blocks. US-guided

Table 4. Multiple logistic regression analysis for possible outcome predictors for injection effectiveness at follow-up

\begin{tabular}{lccc}
\hline \multicolumn{1}{c}{ Factor } & OR & $\mathbf{9 5 \%}$ CI & p-value \\
\hline Age & 1.01 & $0.97-1.06$ & 0.65 \\
Gender & 1.03 & $0.46-2.31$ & 0.94 \\
Symptom duration & 0.98 & $0.81-1.18$ & 0.81 \\
No. of injections & 0.59 & $0.30-1.15$ & 0.12 \\
Cause (spinal stenosis or herniated lumbar disc) & 0.56 & $0.18-1.68$ & 0.30 \\
Ultrasound- or fluoroscopy-guided method & 0.97 & $0.45-2.12$ & 0.94 \\
\hline Contrast pattern & 1.02 & $0.35-2.14$ & 0.68 \\
\hline
\end{tabular}

OR, odds ratio; CI, confidence interval. 
approach enables visualization of soft tissues, nerves, blood vessels and others through real-time imaging, it also observes the degree of diffusion for injected medications around nerves and prevent the nerve damages by injection needles [17]. Narouze et al. [4] published a report on two major vessels on the frontal and posterior side of the foramen in 6 patients with a color Doppler during a prospective study on the US-guided nerve root block. A different study reported that more than $20 \%$ (21/95) of the subjects under investigation had either an ascending or deep cervical artery or a large branch within $2 \mathrm{~mm}$ of the needle path for cervical transforaminal procedures [18]. One-third of these vessels were spinal branches that entered the foramen posteriorly, potentially forming a radicular or a segmental feeder vessel to the spinal cord [18].

According to a comparative study of Jee et al. [6] on USguided cervical selective nerve root block and FL-guided cervical transforaminal epidural block, deep cervical artery was observed in posterior intervertebral foramen for 4 patients out of 55 subjects. Deep cervical artery or radiculomedullary was located in the place to be injured by needle in the FL-guided approach. After the injection of contrast agent, intravascular injection was not confirmed in US-guided group and found in 5 subjects in FL-guided group. However, no statistically significant difference was found [6]. In this study, the intravascular injection was not detected in US-guided group, but observed in 3 subjects of the FL-guided group. However, no statistically significant differences were found.

Although the US may assist to avoid intravascular injections, it is not clear if it can help to detect such injections. To avoid this, the study used two methods. First, nonparticular steroid was implemented as the medication.

Tiso et al. [19] recommended small particles of dexamethasone or betamethasone since the particles of methylprednisolone or triamcinolone flocculate may block smaller arteries by forming large coagulations with $100 \mu \mathrm{m}$ or larger in diameters. Derby et al. [20] also reported that the arterial or capillary obstruction should be avoided during the inadvertent injection into the artery due to the size of the dexamethasone sodium phosphate particles, which is one-tenth the size of the red blood cells.

Second, test dose injection technique was applied using local anesthetics, such as lidocaine and others. Smuck et al. [8] recommended $1 \mathrm{~mL}$ of $1 \%$ lidocaine as the anesthetic test dose to prevent intravascular injection during the transforaminal injection procedures. The rate of positive findings during the intravascular injections was $0.56 \%$ during the test dose injections. The $1 \mathrm{~mL}$ of $1 \%$ lidocaine was used as the test dose before the drug injections in this study. The patients were monitored for every special reaction for 1 to 2 minutes after the test dose injections before proceeding to the actual treatment injections. Although positive findings of intravascular injections were not observed, the test dose method was applied in both procedures to prevent possible intravascular injections.

To achieve satisfactory treatment effects of epidural steroid injection, sufficient amount of steroid needs to be administered to anterior epidural space, foraminal stenosis lesion causing corresponding radicular pains. Unlike FL-guided approach, the US-guided approach often manifested by spreading tendency of contrast agents along with nerve roots toward the distal portion. As a result, treatment effects and durations were considered to decrease when compare to FL-guided approach. In this study, radiographic analysis results revealed that contrast agent was observed only in distal portions of 45 subjects $(70.3 \%)$ and in proximal spinal canal and intraspinal epidural space in 19 subjects $(29.7 \%)$ from US-guided group. On the other hand, contrast agent was observed in proximal spinal canal and intraspinal epidural space for all patients of FL-guided group. However, treatment successes were unrelated to radiologic findings. Yamauchi et al. [5] have explained such results with hydrostatic pressures and osmotic effects. Such mechanisms may have led the solutions to be further absorbed into the nerve fiber. In another words, the intracellular fluid flow may have pushed the local anesthetics and steroids towards the central lesion, the site of possible nerve root compressions or inflammations. Second, the differences in viscosity of the drug and contrast could also support the findings. Jee et al. [6] demonstrated that the spreading of the contrast media into the intraforaminal epidural space was observed by comparing the anteroposterior images taken after the contrast media injections and the washout images taken after the drug injections in 25 patients. The observations of the contrast media, Omnipaque and in the intraspinal epidural area post washout, despite its comparatively high viscous nature [21], could be explained by the injected drug $(0.5 \%$ lidocaine 
$1 \mathrm{~mL}+$ dexamethasone $1 \mathrm{mg}$ ). The injected drug, with its low viscosity, may have diluted and further spread the contrast media into the targeted area [6]. Such phenomena may be explained by the proximal delivery of the drug to the lesions for higher efficacy. Based on the results of the above two studies, US-guided approach is anticipated to have the same treatment effects with FL-guided approach.

This study used non-particle steroids as injection medication. In the previous study, cervical selective nerve root block was performed by using dexamethasone and triamcinolone to compare clinical effects, and although more favorable outcome was shown in triamcinolone group, no statistically significant differences were found in patient satisfaction scores during short-term followups [22]. Jee et al. [6] used injection techniques of both FL and US and compared short-term treatment effect when dexamethasone was injected. Pain index and function index improved in both injection methods as compared with prior injection.

This study identified factors that could influence treatment outcomes. Although the authors were unable to find statistically significant results, the successfully rate of treatment was 0.56 times higher in HCD as compared to spinal stenosis. Choi et al. [23] examined prognostic factors of lumbar transforaminal epidural steroid injection in patients with lower radiating pains. They found out that favorable treatment outcomes were shown in patients with herniated intervertebral discs while unfavorable treatment results were shown in patients with failed back surgery syndromes and spondylolisthesis. Slipman and Chow [24] suggested that treatment effects of epidural injections were more outstanding in patients with herniated intervertebral disc than in patients with lumbar or cervical stenosis. They reported that the unfavorable outcomes of stenosis patients are attributable to various factors including circulatory disorders, inflammations, structural nerve root damages, and others involved in stenosis symptoms.

There are some limitations in the study. First, we were unable to exclude several other factors that could affect treatment effects since this study was a retrospective study. Second, intermittent FL was limited to detect the condition of intravascular injections of contrast media as compared to real-time imaging [25]. Therefore, the results of this study are not fully reliable, since the intra- vascular injection rates of contrast agents are low in both approaches used in the study. Prospective randomized long-term studies should be performed in the future to supplement such limitations.

In conclusion, US-guided selective nerve root block enables injection by avoiding blood vessels that could be injured with the use of FL-guided approach and by visualizing nerve roots and major blood vessels in real-time. In terms of treatment effects, insignificant differences were found in 12-month long-term treatment effects, the degree of functional improvements, and patient satisfactions without radiation exposures as compared to the FLguided approach. Therefore, the US-guided approach is recommended.

\section{CONFLICT OF INTEREST}

No potential conflict of interest relevant to this article was reported.

\section{REFERENCES}

1. Slipman CW, Lipetz JS, Jackson HB, Rogers DP, Vresilovic EJ. Therapeutic selective nerve root block in the nonsurgical treatment of atraumatic cervical spondylotic radicular pain: a retrospective analysis with independent clinical review. Arch Phys Med Rehabil 2000;81:741-6.

2. Boswell MV, Hansen HC, Trescot AM, Hirsch JA. Epidural steroids in the management of chronic spinal pain and radiculopathy. Pain Physician 2003;6:319-34.

3. Manchikanti L. Transforaminal lumbar epidural steroid injections. Pain Physician 2000;3:374-98.

4. Narouze SN, Vydyanathan A, Kapural L, Sessler DI, Mekhail N. Ultrasound-guided cervical selective nerve root block: a fluoroscopy-controlled feasibility study. Reg Anesth Pain Med 2009;34:343-8.

5. Yamauchi M, Suzuki D, Niiya T, Honma H, Tachibana $\mathrm{N}$, Watanabe A, et al. Ultrasound-guided cervical nerve root block: spread of solution and clinical effect. Pain Med 2011;12:1190-5.

6. Jee H, Lee JH, Kim J, Park KD, Lee WY, Park Y. Ultrasound-guided selective nerve root block versus fluoroscopy-guided transforaminal block for the treatment of radicular pain in the lower cervical spine: a randomized, blinded, controlled study. Skeletal Ra- 
diol 2013;42:69-78.

7. Galiano K, Obwegeser AA, Bodner G, Freund MC, Gruber $\mathrm{H}$, Maurer $\mathrm{H}$, et al. Ultrasound-guided periradicular injections in the middle to lower cervical spine: an imaging study of a new approach. Reg Anesth Pain Med 2005;30:391-6.

8. Smuck M, Maxwell MD, Kennedy D, Rittenberg JD, Lansberg MG, Plastaras CT. Utility of the anesthetic test dose to avoid catastrophic injury during cervical transforaminal epidural injections. Spine J 2010; 10:857-64.

9. Nahm FS, Lee CJ, Lee SH, Kim TH, Sim WS, Cho HS, et al. Risk of intravascular injection in transforaminal epidural injections. Anaesthesia 2010;65:917-21.

10. Hartrick CT, Kovan JP, Shapiro S. The numeric rating scale for clinical pain measurement: a ratio measure? Pain Pract 2003;3:310-6.

11. Vernon H, Mior S. The neck disability index: a study of reliability and validity. J Manipulative Physiol Ther 1991;14:409-15.

12. Manchikanti L, Singh V, Falco FJ, Cash KA, Pampati V. Lumbar facet joint nerve blocks in managing chronic facet joint pain: one-year follow-up of a randomized, double-blind controlled trial. Clinical Trial NCT00355914. Pain Physician 2008;11:121-32.

13. Baker R, Dreyfuss P, Mercer S, Bogduk N. Cervical transforaminal injection of corticosteroids into a radicular artery: a possible mechanism for spinal cord injury. Pain 2003;103:211-5.

14. Houten JK, Errico TJ. Paraplegia after lumbosacral nerve root block: report of three cases. Spine J 2002; 2:70-5.

15. McLain RF, Fry M, Hecht ST. Transient paralysis associated with epidural steroid injection. J Spinal Disord 1997;10:441-4.

16. Muro K, O'Shaughnessy B, Ganju A. Infarction of the cervical spinal cord following multilevel transforami- nal epidural steroid injection: case report and review of the literature. J Spinal Cord Med 2007;30:385-8.

17. Narouze S, Peng PW. Ultrasound-guided interventional procedures in pain medicine: a review of anatomy, sonoanatomy, and procedures. Part II: axial structures. Reg Anesth Pain Med 2010;35:386-96.

18. Huntoon MA. Anatomy of the cervical intervertebral foramina: vulnerable arteries and ischemic neurologic injuries after transforaminal epidural injections. Pain 2005;117:104-11.

19. Tiso RL, Cutler T, Catania JA, Whalen K. Adverse central nervous system sequelae after selective transforaminal block: the role of corticosteroids. Spine J 2004;4:468-74.

20. Derby R, Lee SH, Date ES, Lee JH, Lee CH. Size and aggregation of corticosteroids used for epidural injections. Pain Med 2008;9:227-34.

21. Furman MB, Mehta AR, Kim RE, Simon JI, Patel R, Lee TS, et al. Injectate volumes needed to reach specific landmarks in lumbar transforaminal epidural injections. PM R 2010;2:625-35.

22. Dreyfuss P, Baker R, Bogduk N. Comparative effectiveness of cervical transforaminal injections with particulate and nonparticulate corticosteroid preparations for cervical radicular pain. Pain Med 2006;7:237-42.

23. Choi BI, Han JM, Kweon TD, Lee YW. The prognostic factors of selective transforaminal epidural block in patients with low back pain. Korean J Pain 2007;20:549.

24. Slipman CW, Chow DW. Therapeutic spinal corticosteroid injections for the management of radiculopathies. Phys Med Rehabil Clin N Am 2002;1:697-711.

25. Smuck M, Fuller BJ, Chiodo A, Benny B, Singaracharlu $\mathrm{B}$, Tong $\mathrm{H}$, et al. Accuracy of intermittent fluoroscopy to detect intravascular injection during transforaminal epidural injections. Spine (Phila Pa 1976) 2008;33:20510. 